\title{
Effects of choice-figure rotation on the visual perception of form'
}

Fifty-six $S$ s responded in a paper-and-pencil figure-cancellation task to 4-by-4 metric figures. Both random and constrained or "Redundancy-I" figures were used with both rotated and nonrotated choice figures. In terms of speed and accuracy of cancellation, perceptual performance with random figures was better than with constrained, and performance with nonrotated choice figures was better than with rotated. A significant interaction of figure type with rotation indicated that the detrimental effects of choice-figure rotation were especially large when imposed on constrained figures. The effect of choice-figure rotation is interpreted as similar to other "noise" effects that make filtering a necessary part of S's task.

Figure rotation has been identified as one of the "transpositional" parameters that affects the visual perception of form (i.e., a parameter that may be changed without affecting either the uncertainty or the shape of a figure; see Michels \& Zusne, 1965). The perceptual performances of both men and animals appear to be improved with the use of rotated figures in tasks that require similarity or dissimilarity judgments (matching responses or discriminations), especially where the judgments are permitted to be based, at least in part, on the cues to sameness or difference provided by the figures' rotations or relative orientations (cf. Arnoult, 1951; 1954; Brown et al, 1962; French, 1953; Hitchcock et al, 1963; Michels et al, 1962).

On the other hand, rotation does not seem to affect ratings of shape for similarity (Small, 1961), geometricity (Zusne \& Michels, 1962), or association value (Goldstein, 1961). Figure rotations apparently do not provide cues for these sorts of judgments.

These findings suggest an obvious question: How does figure rotation affect matching responses (or discriminations) when the rotation cannot be used as a cue to sameness or difference-i.e., when the rotational transposition is irrelevant to the correct judgment of sameness or difference in form? The present study was designed to answer this question.

\section{Method}

Random and constrained "metric figures" were used, both rotated and nonrotated in a paper-and-pencil figure-cancellation task. These shapes are relatively simple, appearing as solidly dark bar graphs on a white background; they have been described in detail elsewhere (Fitts \& Leonard, 1957; Baker \& Alluisi, 1962).

The metric figures used in the present study were constructed on the basis of an underlying 4-by-4 cell matrix. The random figures were constructed by random sampling of column heights; i.e., by random sampling from the population of $4{ }^{4}$ or 256 random 4 -column metric figures. The constrained or "Redundancy-I" figures were constructed by sampling column heights at random, but with the constraint that each of the four column heights appeared once and only once in each figure; i.e., by random sampling from the population of 4 ! or 24 constrained 4-column metric figures.

A sample of 24 different random metric figures was drawn to serve as target figures in one condition of the study, and the 24 possible constrained figures were used as targets in another condition. These two conditions, the use of random versus constrained or "Redundancy-I" figures, constituted one dimension of the study, rotation versus nonrotation of the choice figures constituted a second dimension of the study, factorial to the first.

The stimulus figures were constructed by using small solid squares (approximately $0.04 \mathrm{~cm}$ sq.) produced by a special key on an electric typewriter. The figures were typed directly onto spirit masters and reproduced by spirit duplication as purple-colored forms on one side of 8-1/2 $\times 11$ in. sheets of white paper. The 24 sampled target figures of a given type appeared on each of four different sheets-two sheets (or 48 problems) of rotated choice figures and two of nonrotated figures. The two sheets of a given kind of target and condition of choice-figure rotation were then stapled together to form a subtest, and four subtests were stapled together to form a test. The order of subtests was balanced across test booklets, with $1 / 4$ of the booklets arranged according to each of four orders: 1234, 3412, 2143, and 4321 , where 1 and 2 are random-figure subtests, 3 and 4 are constrained-figure subtests, and the even numbers represent rotatedchoice-figure subtests.

Each sheet consisted of 24 problems arranged in two columns of 12 problems, the columns' being separated by two heavy lines drawn down the center of the page. Each problem consisted of a target figure on the left and three choice figures on the right; 2-1/2 figure widths separated left from right, and choice figures were separated by unit figure widths. The separate problems, or rows, were also separated by unit figure widths.

The first (left-most) and second choice figures were metric figures of the same type as the target figure, whereas the third choice figure was in all cases an open square equal in over-all area to the cell matrix (approximately $0.64 \mathrm{~cm} \mathrm{sq.)}$. The task required $\mathrm{S}$ to look at the target figure on the left of each row and then to cross out either the first or the second choice figure if it was identical to the target, or the open square if neither of the first two choice figures matched the target. A rotated choice figure, if otherwise identical to the target, was to be cancelled as a matching figure.

All target figures were oriented identically at $0-\mathrm{deg}$. rotation, i.e., with base horizontal and bars rising. In the "rotated" condition, choice figures appeared at $0,90,180$, or $270-\mathrm{deg}$. clockwise rotation, determined at random and independently for each figure; all choice figures appeared at 0-deg. rotation in the "nonrotated" condition. Each sheet of 24 problems called for correct responses of eight cancellations in each of the three possible choice positions, but the order was random and different for each sheet.

The tests were administered to 56 students (25 males and 31 females) in the introductory and developmental psychology classes at the University of Louisville; Ss ranged in age from 18 to 41 years, with a median of 23 . None had previously served in any similar study of form perception.

Elapsed time from the beginning of testing was written in 5-sec. intervals on a blackboard in front of the group-testing room by $E$; the digits were large and clearly visible to all Ss. Elapsed time was recorded by each $S$ at the bottom of each page of the test booklet as he completed that page. Thus, each of the 56 completed test booklets consisted of four cancellation responses (two each with rotated and nonrotated choice figures) for each of the 48 target figures (24 random and 24 constrained), or 192 cancellation responses in all, and also of eight elapsed time recordings-one on each of the eight sheets of the test booklet. 
Table 1. Mean Percentage of Incorrect Cancellations (and, in Parentheses, Mean Response Time per Figure Cancellation, in Seconds)

\begin{tabular}{lccc} 
Type of Target & \multicolumn{2}{c}{ Choice-Figure Rotation } & Mean \\
\cline { 2 - 4 } \& Choice Figures & Nonrotated & Rotated & \\
\hline Random & 2.60 & 4.46 & 3.54 \\
& $(2.632)$ & $(3.380)$ & $(3.006)$ \\
& & & \\
Consfrained & 4.98 & 7.81 & 6.40 \\
& $(3.473)$ & $(5.071)$ & $(4.272)$ \\
\hline Mean & 3.79 & 6.15 & 4.98 \\
& $(3.052)$ & $(4.225)$ & $(3.639)$ \\
\hline
\end{tabular}

\section{Results}

The mean percentage of incorrect figure cancellations and the mean response time per cancellation are given for each of the four experimental conditions in Table 1 . The response times (in seconds) are given parenthetically below the error percentages.

Analyses of variance of these data, based on the 3dimensional factorial design of figure type ( $\mathrm{T}$ ) by rotation condition (R) by subject (S), yielded similar results with both errors and response times. In both cases, the differences attributable to figure types, to rotation conditions, and to the $\mathrm{T}-\mathrm{by}-\mathrm{R}$ interaction were statistically significant ( $F$ ratios of $74.5,18.8$, and 26.1, respectively, in the case of the error scores, and 104.5, 112.4, and 34.8 , respectively, in the case of the cancellation times; with df of $1 / 55$ in each case, $p<.01$ ).

The data of Table 1 indicate that performance with random figures was better (fewer errors and briefer response times) than with constrained figures. Performance with nonrotated figures was also better than with rotated choice figures. The interaction reflects the better-than-expected performance obtained with nonrotated random figures, and the worse-than-expected performance yielded by rotated constrained figures.

\section{Discussion}

As was noted in the opening paragraph, the results of a number of studies indicate that perceptual performance is improved with the use of rotated figures, where judgments of similarity or dissimilarity (matching responses or discriminations) are cued or clued by rotations or relative orientations of figures. That is to say, both men and animals will identify quicker and more accurately a "different" figure from a group when that figure is rotated into a different orientation relative to the "same" figures in the group. In such cases, rotation or relative orientation serves as an additional basis for discrimination-figures that are not oriented alike are not the same, even though figures that are oriented alike may still be different.
Rotation was not included to provide a helpful cue or clue to performance in the present study. Indeed, rotation was imposed as an additional source of uncertainty (or variation) that $S$ would have to ignore in making his judgment of sameness or difference of target and choice figures. The data clearly confirm that rotation of the choice figure under these conditions is not an aid to performance; rather, it is detrimental to both speed and accuracy. This is consistent with the interpretation that the rotation serves as a source of undesirable uncertainty or "noise" which S must iilter. It should be possible to compare the detrimental effects of this type of noise (rotation) with other types (random cell noise or contour noise; cf. Fitts \& Leonard, 1957), and thereby to obtain a better understanding of man's ability to filter noise in his visual perception of form.

\section{References}

Amoult, M. D. Measures of shape discrimination performance in a study of the effect of angular orientation of the stimulus. USAF HRRC Res. Note, 1951, No. 51-10.

Amoult, M. D. Shape discrimination as a function of the angular orientation of the stimuli. J. exp. Psychol., 1954, 47, 323-328,

Baker, E. J., \& Alluisi, E. A. Information handling aspects of visual and auditory form perception. J. engng. Psychol., 1962, $1,159-179$.

Brown, D. R., Hitchcock, L. Jr., \& Michels, K. M. Quantitative studies in form perception: An evaluation of the role of selected stimulus parameters in the visual discrimination performance of human subjects. Percept. mot. Skills, 1962, 14, 519-529.

Fitts, P. M. \& Leonard, J. A. Stimulus correlates of visual pattern recognition-a probability approach. Wash. D. C., Off. Naval Res., Final Rep. of Contract Nont-495(02), October, 1957.

French, R. S. The accuracy of discrimination of dot patterns as a function of angular orientation of the stimuli. USAF HRRC Res. Bull., 1953, No. 53-3.

Goldstein, A. G. Spatial orientation as a factor in eliciting associative response to random shapes. Percept. mot. Skills, 1961, 12, 15-25.

Hitchcock, L. Jr., Michels, K. M., \& Brown, D. R. Discrimination learning: Squirrels vs. raccoons. Percept. mot. Skills, 1963 , $16,405-414$.

Michels, K. M., Pittman, G. G., Hitchcock, L. Jr., \& Brown, D. R. Visual discrimination: Tree squirrels and quantified stimulus dimensions. Percept. mot. Skills, 1962, 15, 443-450.

Michels, K. M., \& Zusne, L. Metrics of visual form. Psychol. Bull., 1965, 63, 74-86.

Small, V. I. Judged similarity of visual forms as functions of selected stimulus dimensions. Unpublished doctoral dissertation, Purdue University, 1961.

\section{Note}

1. Supported in part by the U. S. Army Medical Research and Development Command, Department of the Army, under Research Contract No. DA-49-193-MD-2567, "Behavioral Effects of Infectious Diseases," and based on a senior thesis submitted by the senior author in partial fulfillment of the requirements for the degree, Bachelor of Arts, at the University of Louisville. The research was supervised by the junior author. 